\title{
The Sterols of Growth and Stationary Phases of Aspergillus nidulans Cultures
}

\author{
By JOSEPH L. EVANS AND MICHAEL A. GEALT* \\ Department of Biological Sciences, Drexel University, 32nd and Chestnut Streets, Philadelphia, \\ PA 19104, USA
}

(Received 10 August 1984; revised 18 September 1984)

\begin{abstract}
The accumulation of 4-desmethyl and 4,4-dimethyl sterols, as well as the triterpenoid $\beta$-amyrin, was analysed during both exponential and stationary phases of Aspergillus nidulans growth. Throughout growth, the amount of 4-desmethyl sterol was proportional to the cellular dry weight, while the dimethyl sterols and $\beta$-amyrin stopped accumulating after day 2 . The sterols were found primarily as the free alcohol and not as fatty acid esters, the glycosides, or acyl glycosides. The amount of $\beta$-amyrin in stationary phase cultures was affected by the concentrations of $\mathrm{Mg}^{2+}$ and $\mathrm{Cu}^{2+}$.
\end{abstract}

\section{INTRODUCTION}

The quantitative regulation of sterol production is a complex, but necessary, process for eukaryotes. Aspergillus nidulans produces ergosterol as its major 4-desmethyl sterol and lanosterol as its initial 4,4-dimethyl sterol (Shapiro \& Gealt, 1982). $\beta$-Amyrin is found as an alternative to lanosterol as a cyclization product of squalene (Gealt, 1983). The biosynthetic pathways for the sterols have been described in several fungi (Parks, 1978; Weete, 1980). However, the regulation of the cellular concentration of individual sterols, as well as the molecular form of these sterols (free sterol, steryl ester, steryl glycoside, etc.), remains obscure.

Many fungi, including the Aspergilli, accumulate specific metabolites upon entering the stationary phase of their life cycle. In some cases, such as aflatoxin synthesis in A. flavus, the metabolite is detected only during stationary phase and not during exponential phase (Bennett \& Christensen, 1983). In the case of citric acid production by $A$. niger, synthesis, albeit in reduced amounts, occurs early in the growth cycle, but the major accumulation occurs during stationary phase (Berry et al., 1977). It has been suggested that the sterols may be secondary metabolites in some fungal systems (Demain, 1981; Malik, 1980). Indeed, the accumulation of steryl esters by Saccharomyces cerevisiae is characteristic of stationary phase metabolism (Parks, 1978). Although $A$. nidulans is capable of producing secondary metabolites, e.g. penicillin (Holt \& Macdonald, 1968) and sterigmatocystin (Ishida et al., 1972), we report here that, while $A$. nidulans did not accumulate free 4-desmethyl sterols, free 4,4-dimethyl sterols, free $\beta$-amyrin, or steryl esters as secondary metabolites, comparisons of the accumulation patterns for these products indicated that regulated alterations did occur during the growth cycle.

\section{METHODS}

Organism and growth conditions. A. nidulans FGSC-4 was grown either on YG medium [yeast extract (Difco), $5 \mathrm{~g} \mathrm{I}^{-1}$ : D-glucose, $10 \mathrm{~g} \mathrm{l}^{-1}$ ] or on modified yeast extract/glucose/salts media; trace element stock solution $\left(1 \mathrm{ml}^{-1}\right)$ was utilized in all of the modified media. The stock solution consisted of $\left(1^{-1}\right): 40 \mathrm{mg} \mathrm{Na} \mathrm{N}_{4} \mathrm{O}_{7} .10 \mathrm{H}_{2} \mathrm{O}, 400 \mathrm{mg}$ $\mathrm{CuSO}_{4} .5 \mathrm{H}_{2} \mathrm{O}, 800 \mathrm{mg} \mathrm{FeSO}{ }_{4} .2 \mathrm{H}_{2} \mathrm{O}, 800 \mathrm{mg} \mathrm{Na} 2 \mathrm{MoO}_{4} \cdot 2 \mathrm{H}_{2} \mathrm{O}, 800 \mathrm{mg} \mathrm{MnSO}_{4} . \mathrm{H}_{2} \mathrm{O}$, and $8 \mathrm{~g} \mathrm{ZnSO} 4.7 \mathrm{H}_{2} \mathrm{O}$. Specific medium modifications are described in Results.

Cultures were grown in 2-litre Erlenmeyer flasks containing 11 of medium, or 250-ml Erlenmeyer flasks

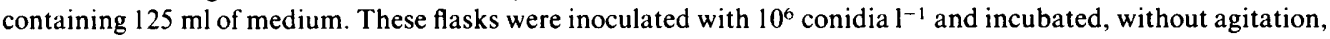


at $30^{\circ} \mathrm{C}$, for periods of 1 to $7 \mathrm{~d}$. After incubation, the cells were collected by vacuum filtration through Miracloth (Calbiochem-Behring, La Jolla, Calif., USA). The lipids were extracted immediately following collection (see below). Culture dry weight was measured for the mass remaining after extraction.

Lipid extraction and purification. The amount of total sterol in the cultures was determined by direct saponification of cell mass in $5 \%$ (w/v) KOH in methanol/water $(9: 1, v / v)$ followed by extraction of neutral lipids with anhydrous ether. Acid hydrolysis of cells prior to saponification according to the method of Gonzalez \& Parks (1977) was utilized to analyse for the presence of water-soluble sterol complexes. The amount of 4-desmethyl sterol was determined by densitometry of TLC using a Hoefer densitometer (Model GS300) coupled with a SpectraPhysics Integrator (Model 4270). The linearity of response was checked by using a set of quantitative authentic ergosterol standards. Care was taken to keep all lipid solutions in the dark to prevent light-induced degradation of ergosterol.

Free sterols and fatty acyl steryl esters were extracted by a modification of the Bligh \& Dyer chloroform/ methanol procedure as described by Kates (1972). After immersion of the cells in the chloroform/methanol $(1: 2$, $\mathrm{v} / \mathrm{v})$ extraction solution, the cells were homogenized for $1 \mathrm{~min}$ at maximum speed in a Sorvall Omni-Mixer. The cells were then extracted for an initial period of $3 \mathrm{~h}$, and then, after recovery by filtration through Miracloth, for a second period of $2 \mathrm{~h}$ with methanol/chloroform/water (10:5:4, by vol.). The extracts were pooled and approximately twice the volume of a $1: 1(\mathrm{v} / \mathrm{v})$ chloroform/water mixture was added to the extract (in a separatory funnel) and the liquids were mixed by inversion. This mixture separated into two phases while standing in the dark overnight at room temperature (approximately $18^{\circ} \mathrm{C}$ ): a chloroform phase containing the majority of lipid material, and a methanol/water phase containing non-lipid material plus some lipids bound into water-soluble complexes, e.g. glycosides. TLC analysis of the methanol/water phase on Silica $60 \mathrm{G}$ used a mobile phase of chloroform/methanol $(9: 1, \mathrm{v} / \mathrm{v})$ (T. A. Salt \& J. H. Adler, personal communication), which yielded an $R_{F}$ of 0.98 for cholesteryl oleate, 0.86 for free ergosterol, 0.60 for acylated steryl glucosides, and $\mathbf{0 . 3 0}$ for steryl glucosides. Additional analysis of the methanol/water for the presence of sterol compounds was performed by hydrolysing the methanol/water solution with acid (after addition of approx. 10 vols $0 \cdot 1 \mathrm{M}-\mathrm{HCl}$ to the solution, followed by heating at $95^{\circ} \mathrm{C}$ for $20 \mathrm{~min}$ ) and/or saponification, followed by extraction with anhydrous ether.

Lipid materials were dried, weighed, redissolved in chloroform and stored at $-20^{\circ} \mathrm{C}$. The sterols and steryl esters were purified from the extract by preparative TLC on Silica Gel $60 \mathrm{G}$ using a solvent system of benzene/ethyl acetate $(8: 2, \mathrm{v} / \mathrm{v})$. TLC plates were examined under long-wave UV to verify the location of fluorescent compounds, e.g. ergosterol. Each lipid fraction was recovered by scraping the silica from the glass plate and then extracting the silica twice with chloroform and once with anhydrous ether. Contaminating silica was removed from the sample by filtration through both a glass fibre filter (Whatman GF/D) and a Nylon-66 filter (Rainin, Inc., Woburn, Mass., USA). The total free sterol was calculated by averaging the quantities recovered from either ether extraction of saponified material or chloroform/methanol extraction of nonsaponified material (plus saponification of the methanol/water phase to recover any complex lipids which partition into this phase). This averaging was justified because the two methods of lipid recovery yielded similar quantitative results.

Sterol analysis. GLC was performed utilizing a Perkin Elmer Sigma 3B chromatograph equipped with a flame ionization detector and linked to a Sigma 15 data station. Column packings of $1 \% \mathrm{XE}-60$ (at $220^{\circ} \mathrm{C}$ ) and $3 \% \mathrm{QF}-1$ (at $230^{\circ} \mathrm{C}$ ) were used for analysis. HPLC was performed on a Perkin Elmer Series 3 HPLC using a reverse phase C-18 column (Zorbax ODS, DuPont). Material was detected by UV absorption with a variable wavelength detector (Perkin Elmer LC-75 or LC-85 equipped with an autocontroller to perform wavelength scans). The free sterols were analysed using a mobile phase of acetonitrile/isopropanol $(4: 1, \mathrm{v} / \mathrm{v})$. These sterols were characterized by their mobility relative to cholesterol (relative retention time, RRT, $=1.00$ on GLC and $\alpha_{c}=1.00$ on HPLC). The quantity of free sterol was detemined by GLC using authentic ergosterol as an external standard. Steryl esters were characterized by their HPLC mobility relative to cholesteryl oleate $\left(\alpha_{c o}=1.00\right.$ on HPLC) using a mobile phase of acetonitrile/isopropanol $(3: 2 . v / v)$. The steryl esters were quantified by HPLC, utilizing authentic ergosteryl oleate as external standard. Authentic standards had the following relative mobilities: ergosteryl oleate, $\alpha_{c o}=0.79$; cholesteryl linoleate, $\alpha_{c o}=0.80$; and cholesteryl linolenate, $\alpha_{c o}=0.64$.

Cholesterol, ergosterol, lanosterol, and 24-dihydrolanosterol were purchased from Supelco, Inc. (Bellefonte, PA). $\beta$-Amyrin was a gift of Dr W. R. Nes (Drexel University) and ergosteryl oleate, cholesteryl oleate, cholesteryl linoleate, and cholesteryl linolenate were gifts of $\mathrm{Dr} \mathrm{J}$. T. Billheimer (Drexel University).

\section{RESULTS}

As reported previously for shaking cultures (Shapiro \& Gealt, 1982), ergosterol (GLC RRT = 1.21 on QF-1 or 1.31 on XE-60) was the major free 4-desmethyl sterol of $A$. nidulans, accounting for $86.0 \%$ to $92.5 \%$ of the total 4 -desmethyl fraction. In static cultures three other free 
4-desmethyl components were observed by GLC on QF-1, with RRTs of 1.07, 1.48, and 1.65. These minor components have not, as yet, been further characterized. Five components with RRTs (on QF-1) of 1.00, 1.29, 1.44, 1.55 and 1.75 were obtained from the 4,4-dimethyl fraction. The component with RRT $=1.55$ has been identified as lanosterol and that with RRT $=1.75$ as $\beta$-amyrin. The other components, which are present in only minor quantities, have not been subjected to additional analyses, although the component with RRT $=1.44$ has a similar retention time to authentic 24-dihydrolanosterol $(R R T=1.43)$ and is tentatively identified as that compound. The identities of ergosterol, lanosterol and $\beta$-amyrin have been confirmed by HPLC, mass spectral analysis, and proton NMR (Shapiro \& Gealt, 1982; Gealt, 1983).

$A$. nidulans cultures grown in YG for periods of 1 to $7 \mathrm{~d}$ were collected and the lipids were extracted. Colonies were completely submerged on day 1 , but had formed a surface mat by day 3 ; no conidiation was observed, even by day 7 . The hyphal residue after extraction was used to measure the dry weight of the cultures (Table 1). The $\mathrm{pH}$ of the medium remained relatively constant during the entire culture period, with a decrease from $\mathrm{pH} 6$ to $\mathrm{pH} 5.5$ during the first $24 \mathrm{~h}$ of culture, then remaining at approximately $\mathrm{pH} 5 \cdot 5$ through 7 days.

The total sterol extracted from the $\boldsymbol{A}$. nidulans hyphal mat increased steadily with culture age. The amounts of each of the three major 4-desmethyl sterols and the 4,4-dimethyl sterols obtained as free sterols during the culture period from day 1 to day 7 are indicated in Table 1. Comparison of the 4-desmethyl content with the amount of 4,4-dimethyl sterol supported the conclusion that, following day 2, most of the free sterol was found as the 4-desmethyl compound (Fig. 1), with ergosterol accounting for the vast majority of this fraction. No major change in total free sterol, or total 4-desmethyl sterol content was observed between days 1 and 7, although a general decreasing trend was noted.

The amount of $\beta$-amyrin in stationary phase $A$. nidulans (6-d cultures) was dependent on the growth period, and on the trace element content of the culture medium. The effect of cations on the synthesis of $\beta$-amyrin was analysed with a series of modifications to create media containing various concentrations of added trace elements. The addition of $\mathrm{Mg}^{2+}$ (either $1.8 \mathrm{~mm}$ $\mathrm{MgSO}_{4} .7 \mathrm{H}_{2} \mathrm{O}$ or $2 \cdot 2 \mathrm{mM}-\mathrm{MgCl}_{2} \cdot 6 \mathrm{H}_{2} \mathrm{O}$ ) to the basal $\mathrm{YG}$ medium which contained approximately $60 \mu \mathrm{M}-\mathrm{Mg}^{2+}$ increased the production of $\beta$-amyrin from a level of $0.04 \pm 0.01 \mathrm{mg}$ (g dry weight) $)^{-1}$ to $0.11 \pm 0.02 \mathrm{mg}$ (g dry weight) ${ }^{-1}$ for $\mathrm{MgSO}_{4} .7 \mathrm{H}_{2} \mathrm{O}$ and $0.22 \pm 0.06 \mathrm{mg}$ (g dry weight) $)^{-1}$ for $\mathrm{MgCl}_{2} \cdot 6 \mathrm{H}_{2} \mathrm{O}$. The omission of the $\mathrm{Cu}^{2+}$ from $\mathrm{MgSO}_{4}$-supplemented trace elements resulted in the lack of any detectable $\beta$-amyrin in cell extracts, while the omission of $\mathrm{Mn}^{2+}$ from the $\mathrm{MgSO}_{4}$-supplemented trace elements marginally decreased the basal level to 0.02 $\pm 0.01 \mathrm{mg}(\mathrm{g} \text { dry weight })^{-1}$. [In yeast extract medium the concentration of $\mathrm{Cu}^{2+}$ is approximately $8 \mathrm{nM}$ and that of $\mathrm{Mn}^{2+}$ is approximately $4 \mathrm{nM}$.] The medium supplemented with $\mathrm{MgCl}_{2} .6 \mathrm{H}_{2} \mathrm{O}$ showed an increase in ergosterol content $[4.61 \pm 0.64$ vs $2.77 \pm 0.79 \mathrm{mg}$ ( $\mathrm{g}$ dry weight $)^{-1}$ ] while none of the other alterations to the medium significantly affected the level of this sterol.

The sterols of $\boldsymbol{A}$. nidulans were present almost totally in the free sterol form during both exponential and stationary phases. The amount of sterol extracted from saponified cells after acid treatment of the culture, compared with the amount obtained without acid treatment, suggested that the cellular sterols were available for extraction without the prior hydrolysis of non-saponifiable bonds to sugars, proteins and lipids. The amount of 4-desmethyl sterol extracted from cultures grown for various periods was as follows: $1.5 \mathrm{~d}, 68.2 \mu \mathrm{g}(\mathrm{mg} \mathrm{lipid})^{-1}$ for both acid-treated and non-acid-treated cells; $3.0 \mathrm{~d}, 87.3 \mu \mathrm{g}$ (mg lipid) ${ }^{-1}$ for acid-treated and $85.6 \mu \mathrm{g}$ (mg lipid) ${ }^{-1}$ for non-acid-treated cells; $5.0 \mathrm{~d}, 83.9 \mu \mathrm{g}$ (mg lipids) ${ }^{-1}$ for acid-treated and $102 \cdot 1 \mu \mathrm{g}$ (mg lipid) ${ }^{-1}$ for non-acid-treated cells. [Each value is the mean of 4-desmethyl sterol recovered from two separate experiments.] The scarcity of complex sterol components was confirmed by TLC examination of both the chloroform portion of chloroform/methanol cell extracts for steryl esters and acyl steryl glycosides, and the methanol portion for steryl glycosides. The methanol layer, which would contain any steryl glycosides, was dried, acid hydrolysed, and analysed. No bands were observed that co-migrated with authentic ergosterol; GLC analysis of materials extracted from this 4-desmethyl region of the TLC plate also confirmed an absence of sterols. The chloroform layer contained only a small amount of material which co-migrated with 


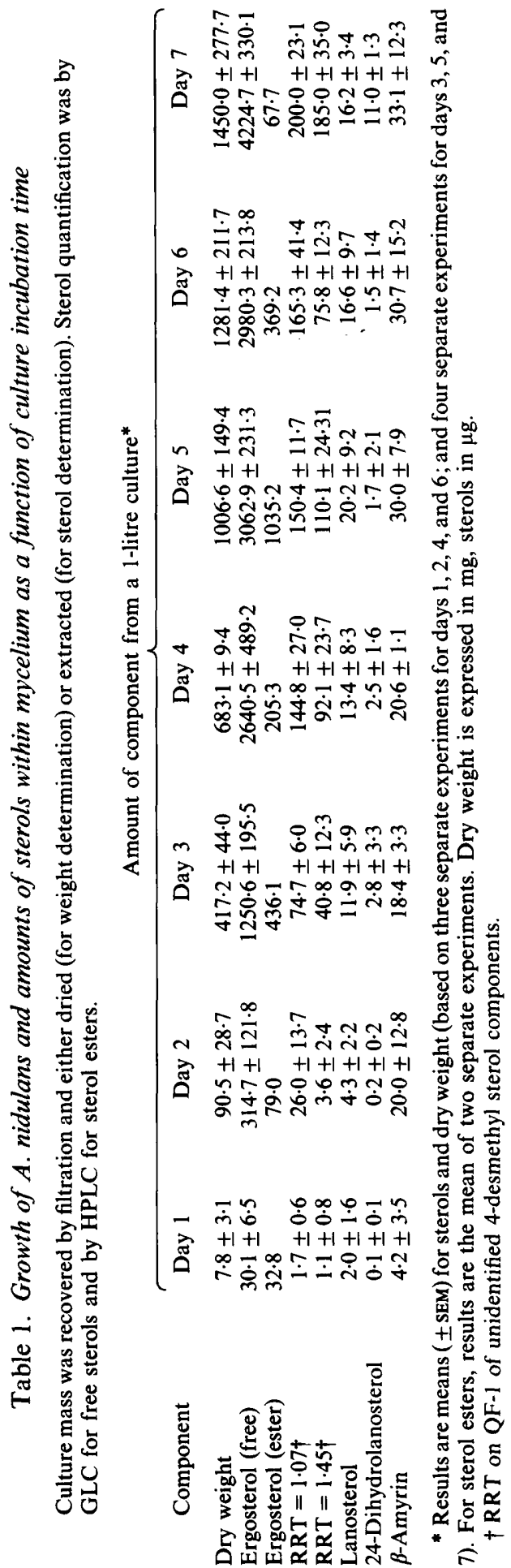




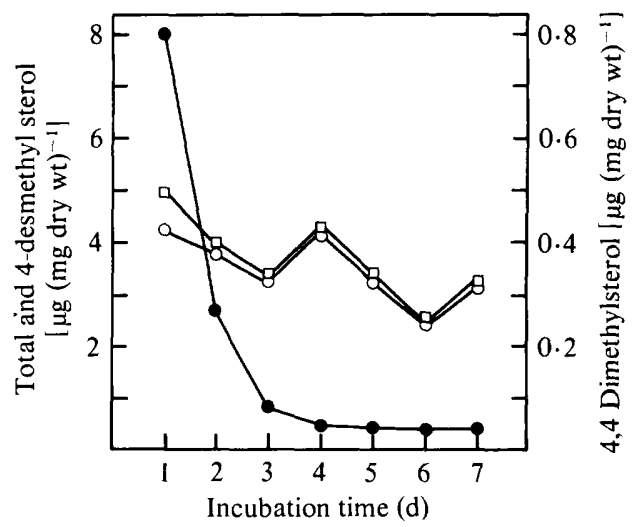

Fig. 1. Amount of sterol extracted from $A$. nidulans cultures grown for 1 to $7 \mathrm{~d}$. $\square$, Total free sterol; $\bigcirc$, free 4-desmethyl sterol; $O$, free 4,4-dimethyl sterol. Each curve represents data derived from daily means for individual sterols and dry weights reported in Table 1.

authentic acyl steryl glycoside. Densitometer comparisons indicated that this band contained approximately $6 \%$ of the material observed in the 4-desmethyl sterol band.

A more prominent band was observed by TLC which co-migrated with authentic steryl esters. Analysis of the steryl esters of $\boldsymbol{A}$. nidulans indicated that the proportion of ergosteryl esters, compared with free ergosterol, decreased slightly with the age of the culture (Table 1). Using the HPLC identification method of Billheimer et al. (1983), we determined that the major ester component was ergosteryl linoleate $\left(\alpha_{\mathrm{co}}=0.62\right)$, representing at least $50 \%$ of the ergosteryl esters on days 2 to 6 . Other esters included ergosteryl oleate $\left(\alpha_{\mathrm{co}}=0.80\right)$ and ergosteryl linolenate $\left(\alpha_{c o}=0.42\right)$. Two minor components were identified as ergosteryl stearate $\left(\alpha_{c o}=1.06\right)$ and lanosteryl linolenate $\left(\alpha_{\mathrm{co}}=0.55\right)$. The relative proportions of the individual sterols were the same in cultures extracted days 1 to 6 .

\section{DISCUSSION}

The sterol composition of $\boldsymbol{A}$. nidulans was very consistent throughout the entire $7 \mathrm{~d}$ growth period, with the exception that 4,4-dimethyl sterols, which are early cyclized precursors of ergosterol, ceased to accumulate after $2 \mathrm{~d}$ growth. All 4-desmethyl components accumulated in parallel with the mass of the culture, indicating that a homeostatic regulatory process is probably involved. The disparity in accumulation patterns between dimethyl and desmethyl sterols suggests that the demethylation of precursors does not appear to be a critical rate-limiting process.

Several patterns of fungal sterol content have been observed as a function of culture age, including the accumulation of ergosterol in Saccharomyces carlsbergensis (Shcheglov \& Anisimov, 1979), the accumulation of ergosteryl esters in S. cerevisiae (Parks, 1978), a production pattern mimicking the growth curve in Penicillium atrovenetum (Van Etten \& Gottlieb, 1965), and a complex pattern of increases and decreases during the growth of Neurospora crassa (Elliott et al., 1974). It should be noted that the fungi of family Pythiaceae do not contain sterols in vegetative cells, although they do require them during the reproductive process (Hendrix, 1970). There does not appear to be any general pattern for developmental regulation of this important membrane lipid component in the fungi.

Interestingly, in $A$. nidulans the sterol in both stationary and exponential growth phases was primarily in the form of free sterol and not the steryl ester. These results are different from those observed in S. cerevisiae (Bailey \& Parks, 1975; Taylor \& Parks, 1978; Taketani et al., 1978) and Phycomyces blakesleeanus (Bartlett \& Mercer, 1974), in which there was a large accumulation of steryl esters. A. nidulans showed a pronounced decrease in the ratio of esterified to free sterol during the first $7 \mathrm{~d}$ of incubation. We have also found that Penicillium urticae does not accumulate steryl esters during growth periods up to $10 \mathrm{~d}$ (Buchanan et al., 1983). These esters 
are usually thought to be storage materials, rather than membrane components. The relatively low levels of steryl esters in $A$. nidulans and $P$. urticae suggest that these fungi are unable to form the esters efficiently or are very efficient at hydrolysing the ester bond, and, as a consequence, maintain their sterols primarily as the free alcohol. The lack of demonstrable steryl glycosides and only the slight amount of acyl steryl glycosides suggest that newly synthesized sterols are probably incorporated into new membrane material and that a subsequent reduction in sterol production occurs when the culture enters stationary phase.

The effects of cations on the accumulation of $\beta$-amyrin suggest that the quantity of material produced was dependent on the overall physiology of the cells. These effects are similar to those noted by Weinberg (1977) and Berry et al. (1977), who observed that the presence of trace elements influences the production of many cellular metabolites of fungi. The ability to synthesize a compound such as $\beta$-amyrin, which presumably is not required for the survival of the organism, is not only an expression of the genetic capability but is also dependent on the characteristics of the environment. It is through the exploration of the physiological capabilities of $\boldsymbol{A}$. nidulans, utilizing the current extensive level of genetic technology available for this organism, that we can investigate the regulation of sterol concentration by this lower eukaryote.

We would like to thank Drs Robert Buchanan and John Adler for their kind encouragement and helpful discussions. The technical assistance of Ms Josephine Landrey, Ms Theresa Lindley and Mr Michael Moclock is gratefully acknowledged.

\section{REFERENCES}

Bailey, R. B. \& Parks, L. W. (1975). Yeast sterol esters and their relationship to the growth of yeast. Journal of Bacteriology 124, 606-612.

BARTLETT, K. \& MERCER, E. I. (1974). Variations in the levels and composition of the sterols and sterol esters of Phycomyces blakesleeanus with age of culture. Phytochemistry 13, 1115-1121.

Bennett, J. W. \& Christensen, S. B. (1983). New perspectives on aflatoxin biosynthesis. Advances in Applied Microbiology 29, 53-92.

BerRy, D. R., Chmiel, A. \& Al Obaidi, Z. (1977). Citric acid production by $A$. niger. In Genetics and Physiology of Aspergillus, pp. 405-426. Edited by J. E. Smith \& J. A. Pateman. New York: Academic Press.

Billheimer, J. T., Avart, S. \& Milani, B. (1983). Separation of steryl esters by reversed-phase liquid chromatography. Journal of Lipid Research 24, 16461651.

Buchanan, R. L., Harry, M. A. \& Gealt, M. A. (1983). Caffeine inhibition of sterigmatocystin, citrinin, and patulin production. Journal of Food Science 48, 1226-1228.

Demain, A. L. (1981). Industrial microbiology. Science 214, 987-995.

Elliott, C. G., Knights, B. A. \& Freeland, J. A. (1974). Sterols of Neurospora crassa and the pattern of their binding during the growth cycle. Biochimica et biophysica acta 360, 339-347.

GEALT, M. A. (1983). Isolation of $\beta$-amyrin from the fungus Aspergillus nidulans. Journal of General Microbiology 129, 543-546.

Gonzales, R. A. \& Parks, L. W. (1977). Acidlabilization of sterols for extraction from yeast. Biochimica et biophysica acta 489, 507-509.

HENDRIX, J. W. (1970). Sterols in growth and reproduction of fungi. Annual Review of Phytopathology 8 , 111-130.
Holt, G. \& Macdonald, K. D. (1968). Penicillin production and its mode of inheritance in Aspergillus nidulans. Antonie van Leeuwenhoek 34, 409-416.

Ishida, M., HaMASAKI, T. \& HATSUdA, Y. (1972). A new metabolite from Aspergillus nidulans. Agricultural and Biological Chemistry 36, 1847-1848.

KATES, M. (1972). Techniques in Lipidology. Amsterdam: North Holland.

MaLIK, V. S. (1980). Microbial secondary metabolism. Trends in Biochemical Science 5, 68-72.

PARKS, L. W. (1978). Metabolism of sterols in yeast. CRC Critical Reviews in Microbiology 6, 301-341.

ShapIRo, B. E. \& Gealt, M. A. (1982). Ergosterol and lanosterol from Aspergillus nidulans. Journal of General Microbiology 128, 1053-1056.

ShCheglov, V. V. \& Anisimov, M. M. (1979). Change in the biosynthesis of sterols and fatty acids during growth of a culture of Saccharomyces carlsbergensis. Izvestiya Akademii Nauk SSSR, Seriya Biologicheskaya No. 3, 462-466.

Taketani, S., NAGaI, J. \& Katsuki, H. (1978). Quantitative aspects of free and esterified sterols in Saccharomyces cerevisiae under various conditions. Biochimica et biophysica acta 528, 416-423.

TAYlOR, F. R. \& Parks, L. W. (1978). Metabolic interconversion of free sterols and steryl esters in Saccharomyces cerevisiae. Journal of Bacteriology 136, 531-537.

Van Etten, J. L. \& Gottlieb, D. (1965). Biochemical changes during the growth of fungi. II. Ergosterol and fatty acids of Penicillium atrovenetum. Journal of Bacteriology 80, 409-414.

WEETE, J. D. (1980). Lipid Biochemistry of Fungi and Other Organisms. New York: Plenum Press.

WeINBERG, E. (1977). Minerals and Microorganisms. New York: Dekker. 